\title{
Local and regional contributions to fine particulate matter in the 18 cities of Sichuan Basin, southwestern China
}

Xue Qiao et al.

Correspondence to: Qi Ying (qying@civil.tamu.edu) and Hongliang Zhang (hlzhang@1su.edu)

The copyright of individual parts of the supplement might differ from the CC BY 4.0 License. 
26 Table S1. The area, population, and economic development, and the concentrations of $\mathrm{PM}_{2.5}$ and

$27 \mathrm{O}_{3}$ of the 18 prefectural cities in the SCB in 2015.

\begin{tabular}{|c|c|c|c|c|c|c|c|}
\hline $\begin{array}{c}\text { Region } \\
\text { ID }\end{array}$ & City ID & City & $\begin{array}{l}\text { Population } \\
(\times \mathbf{1 0 0 0})\end{array}$ & $\begin{array}{l}\text { Area } \\
\left(\mathbf{k m}^{2}\right)\end{array}$ & $\begin{array}{c}\text { GDP } \\
(\times 100 \\
\text { million } \\
\text { RMB }) \\
\end{array}$ & $\begin{array}{c}\text { Annual } \\
\text { PM}_{2.5} \\
\left(\mu \mathrm{g} \mathrm{m}^{-3}\right)^{\#}\end{array}$ & $\begin{array}{c}\text { Annual } \\
\text { PM}_{2.5} \\
\left(\mu \mathrm{g} \mathrm{m}^{-3}\right)^{*}\end{array}$ \\
\hline R1 \& R5 & 1 & Chongqing & 33720 & 82400 & 15717.3 & 57 & 53 \\
\hline \multirow{5}{*}{$\mathbf{R} 2$} & 2 & Bazhong & 3795 & 12293 & 501.34 & & 35 \\
\hline & 3 & Dazhou & 6828 & 16582 & 1350.76 & & 59 \\
\hline & 4 & Guangyuan & 3053 & 16311 & 605.43 & & 21 \\
\hline & 5 & Guang"an & 4674 & 6341 & 1005.61 & & 43 \\
\hline & 6 & Nanchong & 7423 & 12477 & 1516.2 & 61 & 58 \\
\hline \multirow{11}{*}{ R3 } & 7 & Deyang & 3900 & 5910 & 1605.06 & 53 & 53 \\
\hline & 8 & Leshan & 3538 & 12723 & 1301.23 & & 55 \\
\hline & 9 & Luzhou & 5057 & 12236 & 1353.41 & 62 & 59 \\
\hline & 10 & Meishan & 3491 & 7140 & 1029.86 & & 60 \\
\hline & 11 & Mianyang & 5455 & 20248 & 1700.33 & 47 & 45 \\
\hline & 12 & Neijiang & 4204 & 5385 & 1198.58 & & 60 \\
\hline & 13 & Suining & 3788 & 5323 & 915.81 & & 49 \\
\hline & 14 & Ya'an & 1549 & 15046 & 502.58 & & 34 \\
\hline & 15 & Yibin & 5521 & 13266 & 1525.9 & 58 & 56 \\
\hline & 16 & Zigong & 3275 & 4381 & 1143.11 & 74 & 72 \\
\hline & 17 & Ziyang & 5037 & 7960 & 1270.38 & & 40 \\
\hline R4 & 18 & Chengdu & 12281 & 12119 & 10801.2 & 64 & 61 \\
\hline
\end{tabular}

29 \#data source: China Statistical Yearbook 2016, http://www.stats.gov.cn/tjsj/ndsj/2018/indexeh.htm;

30 *calculated by using the data published on the air quality data releasing platform, Ministry of 31 Ecology and Environment of the People's Republic of China. http://106.37.208.233:20035/. 
Table S2. Predicted contributions from different regions to $\mathrm{PM}_{2.5}$ in the 18 city centers of the SCB in the summer.

\begin{tabular}{|c|c|c|c|c|c|c|c|c|c|c|c|c|c|c|c|c|}
\hline \multirow{2}{*}{$\begin{array}{l}\text { Region } \\
\text { ID }\end{array}$} & \multirow[b]{2}{*}{ City } & \multirow{2}{*}{$\begin{array}{c}\mathbf{P M}_{2.5} \\
\left(\mu \mathrm{g} \mathrm{m}^{-3}\right)\end{array}$} & \multicolumn{14}{|c|}{ Percentage contributions from each region, SOA, and others* $(\%)$} \\
\hline & & & $\mathbf{R 1}$ & $\mathbf{R 2}$ & $\mathbf{R 3}$ & $\mathbf{R 4}$ & $\mathbf{R 5}$ & $\begin{array}{c}\text { Within } \\
\text { SCB }\end{array}$ & R6 & $\mathbf{R} 7$ & $\mathbf{R 8}$ & $\mathbf{R 9}$ & $\begin{array}{c}\text { Outside } \\
\text { SCB }\end{array}$ & SOA & Others & Non-local ${ }^{\#}$ \\
\hline $\mathbf{R 1}$ & Chongqing & 64 & 71.2 & 1.3 & 0.9 & 0.1 & 0.6 & 74.1 & 1.8 & 8.5 & 0.0 & 0.5 & 10.8 & 8.8 & 6.3 & 13.7 \\
\hline \multirow{5}{*}{$\mathbf{R 2}$} & Bazhong & 22 & 1.1 & 37.3 & 1.0 & 0.5 & 1.5 & 41.4 & 0.6 & 26.1 & 0.0 & 1.2 & 27.9 & 22.2 & 8.4 & 32.0 \\
\hline & Dazhou & 29 & 0.6 & 45.9 & 0.4 & 0.1 & 3.6 & 50.6 & 0.6 & 23.1 & 0.0 & 1.0 & 24.7 & 16.7 & 8.1 & 29.4 \\
\hline & Guangan & 31 & 5.8 & 45.6 & 0.7 & 0.2 & 2.9 & 55.2 & 1.5 & 18.1 & 0.0 & 1.0 & 20.6 & 16.2 & 8.1 & 30.2 \\
\hline & Guangyuan & 20 & 2.0 & 46.2 & 2.0 & 0.9 & 0.7 & 51.8 & 0.6 & 18.5 & 0.0 & 1.4 & 20.5 & 18.3 & 9.4 & 26.1 \\
\hline & Nanchong & 33 & 5.8 & 52.9 & 1.2 & 0.3 & 1.3 & 61.5 & 1.1 & 14.7 & 0.0 & 0.8 & 16.6 & 15.1 & 6.8 & 25.2 \\
\hline \multirow{11}{*}{$\mathbf{R 3}$} & Deyang & 40 & 3.0 & 1.7 & 57.8 & 13.3 & 0.3 & 76.1 & 0.5 & 6.7 & 0.1 & 0.6 & 7.9 & 9.9 & 6.2 & 26.2 \\
\hline & Leshan & 30 & 3.3 & 0.9 & 53.8 & 6.6 & 0.2 & 64.8 & 1.1 & 7.3 & 0.1 & 0.7 & 9.2 & 20.0 & 6.2 & 20.2 \\
\hline & Luzhou & 41 & 13.0 & 1.0 & 51.9 & 0.5 & 0.4 & 66.8 & 2.8 & 8.6 & 0.0 & 0.6 & 12 & 15.2 & 6.0 & 26.9 \\
\hline & Meishan & 38 & 3.4 & 0.9 & 38.9 & 27.5 & 0.2 & 70.9 & 0.8 & 6.8 & 0.1 & 0.6 & 8.3 & 15.0 & 5.8 & 40.3 \\
\hline & Mianyang & 31 & 3.6 & 3.5 & 59.8 & 2.7 & 0.5 & 70.1 & 0.7 & 9.3 & 0.0 & 0.8 & 10.8 & 12.3 & 6.9 & 21.1 \\
\hline & Neijiang & 39 & 14.2 & 1.7 & 49.9 & 0.9 & 0.4 & 67.1 & 1.9 & 9.3 & 0.1 & 0.6 & 11.9 & 14.9 & 6.1 & 29.1 \\
\hline & Suining & 25 & 16.4 & 21.1 & 11.5 & 0.4 & 1.4 & 50.8 & 1.9 & 18.4 & 0.0 & 1.0 & 21.3 & 20.0 & 7.9 & 60.6 \\
\hline & Ya'an & 14 & 4.2 & 1.1 & 36.1 & 10.2 & 0.3 & 51.9 & 1.0 & 9.6 & 0.1 & 1.2 & 11.9 & 28.2 & 8.0 & 27.7 \\
\hline & Yibin & 37 & 4.2 & 0.8 & 58.8 & 1.4 & 0.3 & 65.5 & 2.0 & 7.1 & 0.1 & 0.6 & 9.8 & 18.4 & 6.2 & 16.5 \\
\hline & Zigong & 45 & 7.9 & 1.1 & 60.0 & 0.9 & 0.3 & 70.2 & 1.7 & 7.5 & 0.0 & 0.5 & 9.7 & 14.0 & 6.0 & 19.9 \\
\hline & Ziyang & 33 & 8.8 & 2.1 & 48.0 & 5.7 & 0.5 & 65.1 & 1.5 & 10.2 & 0.0 & 0.7 & 12.4 & 16.2 & 6.3 & 29.5 \\
\hline R4 & Chengdu & 44 & 2.5 & 1.1 & 13.6 & 60.0 & 0.3 & 77.5 & 0.6 & 5.8 & 0.1 & 0.6 & 7.1 & 9.6 & 6.0 & 24.6 \\
\hline
\end{tabular}

*Others include IC, BC, windblown dust, and sea salt.

"Non-local=Within SCB + Outside SCB - Local 
Table S3. Source-region contributions to the $\mathrm{NH}_{4}{ }^{+}, \mathrm{NO}_{3}{ }^{-}$, and $\mathrm{SO}_{4}{ }^{2-}$ of $\mathrm{PM}_{2.5}$ in each city center of the SCB in the summer.

\begin{tabular}{|c|c|c|c|c|c|c|c|c|c|c|c|c|c|c|c|c|c|c|c|}
\hline \multirow{3}{*}{$\begin{array}{c}\text { Region } \\
\text { ID }\end{array}$} & \multirow{3}{*}{ Cities } & \multicolumn{18}{|c|}{ Source-region contributions $(\%)$} \\
\hline & & \multicolumn{6}{|c|}{$\mathrm{NH}_{4}{ }^{+}$} & \multicolumn{6}{|c|}{$\mathrm{NO}_{3}^{-}$} & \multicolumn{6}{|c|}{$\mathrm{SO}_{4}{ }^{2-}$} \\
\hline & & Conc* & Local & $\begin{array}{l}\text { Non- } \\
\text { local }\end{array}$ & $\begin{array}{c}\text { Within } \\
\text { SCB }\end{array}$ & $\begin{array}{c}\text { Outside } \\
\text { SCB } \\
\end{array}$ & Others $\$$ & Conc* & Local & $\begin{array}{c}\text { Other } \\
\text { regions }\end{array}$ & $\begin{array}{c}\text { Within } \\
\text { SCB }\end{array}$ & $\begin{array}{c}\text { Outside } \\
\text { SCB } \\
\end{array}$ & Others $\$$ & Conc* & Local & $\begin{array}{l}\text { Non- } \\
\text { local } \\
\end{array}$ & $\begin{array}{c}\text { Within } \\
\text { SCB }\end{array}$ & $\begin{array}{c}\text { Outside } \\
\text { SCB }\end{array}$ & Others $^{\$}$ \\
\hline 1 & Chongqing & 4.0 & 53.4 & 38.2 & 65.5 & 26.1 & 8.4 & 0.8 & 81.1 & 17.9 & 91.8 & 7.2 & 1.0 & 12.6 & 39.7 & 36.6 & 43.4 & 33.0 & 23.7 \\
\hline \multirow{5}{*}{2} & Bazhong & 2.1 & 67.1 & 31.7 & 74.0 & 24.8 & 1.2 & 0.3 & 35.6 & 61.0 & 47.9 & 48.8 & 3.3 & 5.9 & 4.3 & 74.6 & 9.4 & 69.5 & 21.1 \\
\hline & Dazhou & 2.6 & 68.3 & 30.4 & 80.1 & 18.6 & 1.3 & 0.7 & 54.6 & 42.8 & 63.6 & 33.9 & 2.6 & 7.0 & 6.2 & 70.5 & 9.3 & 67.3 & 23.4 \\
\hline & Guang'an & 2.8 & 66.5 & 31.9 & 81.0 & 17.5 & 1.5 & 1.0 & 60.0 & 38.4 & 78.7 & 19.8 & 1.6 & 7.4 & 10.9 & 64.8 & 19.2 & 56.4 & 24.4 \\
\hline & Guangyuan & 1.7 & 71.9 & 26.7 & 78.4 & 20.2 & 1.4 & 0.4 & 60.5 & 37.2 & 72.8 & 25.0 & 2.3 & 4.7 & 8.2 & 67.7 & 18.1 & 57.7 & 24.2 \\
\hline & Nanchong & 2.9 & 74.1 & 24.3 & 84.3 & 14.1 & 1.6 & 1.4 & 72.3 & 26.4 & 84.9 & 13.8 & 1.3 & 7.2 & 14.8 & 62.7 & 25.7 & 51.8 & 22.5 \\
\hline \multirow{11}{*}{3} & Deyang & 3.5 & 75.2 & 22.9 & 91.9 & 6.2 & 1.9 & 2.9 & 64.2 & 35.1 & 94.7 & 4.6 & 0.8 & 7.8 & 26.2 & 51.3 & 49.6 & 28.0 & 22.5 \\
\hline & Leshan & 2.4 & 82.0 & 16.6 & 90.5 & 8.0 & 1.4 & 0.9 & 68.8 & 30.0 & 90.8 & 7.9 & 1.2 & 6.3 & 23.1 & 54.2 & 45.7 & 31.7 & 22.7 \\
\hline & Luzhou & 3.7 & 67.3 & 30.5 & 82.9 & 14.9 & 2.2 & 2.2 & 66.1 & 33.2 & 92.9 & 6.4 & 0.7 & 8.8 & 20.8 & 57.0 & 42.8 & 35.1 & 22.2 \\
\hline & Meishan & 3.8 & 68.6 & 29.9 & 92.6 & 5.9 & 1.6 & 2.4 & 39.2 & 60.1 & 94.1 & 5.2 & 0.7 & 8.7 & 17.5 & 63.8 & 55.2 & 26.1 & 18.8 \\
\hline & Mianyang & 2.6 & 76.2 & 22.1 & 89.5 & 8.7 & 1.8 & 1.6 & 77.2 & 21.9 & 92.0 & 7.0 & 1.0 & 6.2 & 22.7 & 53.9 & 39.3 & 37.3 & 23.4 \\
\hline & Neijiang & 3.3 & 64.9 & 33.0 & 85.0 & 12.9 & 2.1 & 1.8 & 58.1 & 41.0 & 90.6 & 8.5 & 0.9 & 8.2 & 17.9 & 59.5 & 40.2 & 37.3 & 22.6 \\
\hline & Suining & 2.6 & 24.6 & 74.3 & 81.1 & 17.8 & 1.1 & 1.0 & 9.6 & 89.3 & 81.6 & 17.3 & 1.1 & 6.5 & 3.0 & 76.8 & 26.7 & 53.1 & 20.2 \\
\hline & Ya'an & 1.4 & 77.5 & 21.7 & 90.0 & 9.1 & 0.8 & 0.3 & 39.8 & 58.6 & 82.4 & 16.0 & 1.6 & 3.8 & 18.1 & 60.9 & 45.8 & 33.2 & 21.0 \\
\hline & Yibin & 3.1 & 81.6 & 16.6 & 87.6 & 10.6 & 1.8 & 1.6 & 81.9 & 17.3 & 92.4 & 6.7 & 0.9 & 7.8 & 30.6 & 45.8 & 45.0 & 31.4 & 23.6 \\
\hline & Zigong & 3.6 & 74.5 & 23.0 & 86.0 & 11.5 & 2.5 & 2.0 & 75.7 & 23.6 & 93.2 & 6.1 & 0.8 & 8.8 & 26.4 & 50.4 & 44.0 & 32.9 & 23.2 \\
\hline & Ziyang & 3.0 & 76.7 & 22.1 & 89.4 & 9.4 & 1.2 & 1.7 & 53.8 & 45.2 & 89.0 & 10.0 & 1.0 & 7.1 & 16.4 & 62.4 & 39.8 & 39.0 & 21.2 \\
\hline 4 & Chengdu & 3.6 & 46.8 & 49.3 & 85.8 & 10.2 & 4.0 & 1.4 & 69.6 & 29.8 & 95.3 & 4.0 & 0.7 & 9.6 & 37.0 & 43.5 & 58.1 & 22.3 & 19.6 \\
\hline
\end{tabular}


Table S4. Source-region contributions to the $\mathrm{NH}_{4}{ }^{+}, \mathrm{NO}_{3}{ }^{-}$, and $\mathrm{SO}_{4}{ }^{2-}$ of $\mathrm{PM}_{2.5}$ in each city center of the SCB in the winter.

\begin{tabular}{|c|c|c|c|c|c|c|c|c|c|c|c|c|c|c|c|c|c|c|c|}
\hline \multirow{3}{*}{$\begin{array}{c}\text { Region } \\
\text { ID }\end{array}$} & \multirow{3}{*}{ Cities } & \multicolumn{18}{|c|}{ Source-region contributions (\%) } \\
\hline & & \multicolumn{6}{|c|}{$\mathrm{NH}_{4}{ }^{+}$} & \multicolumn{6}{|c|}{$\mathrm{NO}_{3}{ }^{-}$} & \multicolumn{6}{|c|}{$\mathrm{SO}_{4}{ }^{2-}$} \\
\hline & & Conc* & Local & $\begin{array}{l}\text { Non- } \\
\text { local }\end{array}$ & $\begin{array}{c}\text { Within } \\
\text { SCB }\end{array}$ & $\begin{array}{c}\text { Outside } \\
\text { SCB }\end{array}$ & Others $\$$ & Conc* & Local & $\begin{array}{l}\text { Other } \\
\text { regions }\end{array}$ & $\begin{array}{c}\text { Within } \\
\text { SCB }\end{array}$ & $\begin{array}{c}\text { Outside } \\
\text { SCB }\end{array}$ & Others $\$$ & Conc* & Local & $\begin{array}{l}\text { Non- } \\
\text { local }\end{array}$ & $\begin{array}{l}\text { Within } \\
\text { SCB }\end{array}$ & $\begin{array}{c}\text { Outside } \\
\text { SCB }\end{array}$ & Others $^{\$}$ \\
\hline 1 & Chongqing & 14.7 & 48.7 & 44.3 & 69.6 & 23.5 & 7.0 & 11.0 & 61.8 & 36.8 & 79.7 & 18.9 & 1.4 & 40.9 & 46.0 & 44.2 & 58.4 & 31.8 & 9.8 \\
\hline \multirow{5}{*}{2} & Bazhong & 6.4 & 52.5 & 45.1 & 62.0 & 35.7 & 2.3 & 6.1 & 24.0 & 72.7 & 39.0 & 57.7 & 3.3 & 13.9 & 8.0 & 81.0 & 21.0 & 68.1 & 11.0 \\
\hline & Dazhou & 8.4 & 58.6 & 39.0 & 73.7 & 24.0 & 2.3 & 8.5 & 35.2 & 62.2 & 52.1 & 45.3 & 2.6 & 17.8 & 11.1 & 77.9 & 23.9 & 65.1 & 11.0 \\
\hline & Guang'an & 10.8 & 60.5 & 36.9 & 78.1 & 19.2 & 2.6 & 12.2 & 47.8 & 50.5 & 69.9 & 28.3 & 1.8 & 21.5 & 16.7 & 73.3 & 36.6 & 53.5 & 10.0 \\
\hline & Guangyuan & 5.9 & 54.6 & 43.0 & 65.0 & 32.6 & 2.5 & 6.8 & 35.7 & 61.5 & 52.3 & 44.8 & 2.9 & 11.8 & 11.1 & 76.2 & 28.9 & 58.4 & 12.8 \\
\hline & Nanchong & 11.4 & 65.9 & 31.5 & 79.7 & 17.7 & 2.7 & 13.5 & 53.2 & 45.1 & 71.0 & 27.3 & 1.8 & 22.1 & 20.3 & 69.7 & 38.4 & 51.6 & 10.0 \\
\hline \multirow{11}{*}{3} & Deyang & 14.9 & 66.0 & 30.8 & 85.8 & 10.9 & 3.3 & 18.5 & 53.0 & 45.7 & 84.5 & 14.3 & 1.3 & 28.2 & 34.0 & 56.2 & 62.9 & 27.3 & 9.8 \\
\hline & Leshan & 13.1 & 72.2 & 25.3 & 87.9 & 9.6 & 2.5 & 14.8 & 54.5 & 44.1 & 86.4 & 12.2 & 1.4 & 26.5 & 30.8 & 60.9 & 66.5 & 25.1 & 8.4 \\
\hline & Luzhou & 15.6 & 60.8 & 36.0 & 80.8 & 16.0 & 3.2 & 16.7 & 54.6 & 44.2 & 82.8 & 16.0 & 1.2 & 33.2 & 30.9 & 61.1 & 58.2 & 33.8 & 8.0 \\
\hline & Meishan & 17.1 & 55.0 & 42.5 & 89.0 & 8.4 & 2.6 & 19.6 & 38.1 & 60.8 & 88.5 & 10.4 & 1.1 & 35.9 & 23.1 & 69.6 & 72.1 & 20.7 & 7.3 \\
\hline & Mianyang & 11.3 & 65.0 & 32.0 & 82.3 & 14.7 & 3.0 & 13.3 & 54.9 & 43.3 & 77.3 & 21.0 & 1.8 & 22.4 & 34.8 & 55.1 & 55.0 & 34.9 & 10.1 \\
\hline & Neijiang & 14.0 & 59.4 & 37.6 & 81.9 & 15.1 & 3.0 & 15.4 & 46.3 & 52.3 & 79.5 & 19.1 & 1.5 & 29.0 & 24.5 & 67.0 & 54.3 & 37.3 & 8.5 \\
\hline & Suining & 11.1 & 19.4 & 78.5 & 77.2 & 20.7 & 2.1 & 12.2 & 10.9 & 87.4 & 68.8 & 29.5 & 1.7 & 22.2 & 7.9 & 83.9 & 39.6 & 52.2 & 8.2 \\
\hline & Ya'an & 9.6 & 64.1 & 33.8 & 85.0 & 13.0 & 2.0 & 9.2 & 40.6 & 57.4 & 80.2 & 17.8 & 2.0 & 21.0 & 25.6 & 66.7 & 63.7 & 28.7 & 7.6 \\
\hline & Yibin & 14.2 & 70.7 & 26.5 & 84.3 & 12.8 & 2.9 & 15.5 & 62.3 & 36.4 & 84.2 & 14.5 & 1.3 & 29.6 & 33.4 & 58.4 & 61.9 & 29.9 & 8.2 \\
\hline & Zigong & 14.5 & 62.6 & 34.0 & 81.7 & 14.9 & 3.5 & 15.2 & 54.6 & 44.0 & 81.4 & 17.2 & 1.4 & 30.9 & 29.7 & 61.4 & 57.3 & 33.9 & 8.9 \\
\hline & Ziyang & 13.4 & 65.3 & 32.4 & 84.8 & 12.9 & 2.4 & 15.7 & 48.5 & 50.0 & 79.4 & 19.1 & 1.5 & 25.9 & 25.7 & 65.5 & 53.9 & 37.3 & 8.8 \\
\hline 4 & Chengdu & 13.8 & 42.9 & 52.5 & 82.7 & 12.7 & 4.6 & 14.4 & 49.3 & 49.5 & 86.0 & 12.8 & 1.2 & 30.6 & 37.9 & 52.5 & 67.4 & 23.0 & 9.6 \\
\hline
\end{tabular}


Table S5. Predicted maximum daily contribution from a given region (MDCs) in SCB city center and the corresponding $\mathrm{PM}_{2.5}$ concentrations in the 47 city center on the same day. Only summer data are included in this table. The units are $\mu \mathrm{g} \mathrm{m}^{-3}$. The numbers in the bold present the contributions 48 due to local emissions or that from R7.

\begin{tabular}{|c|c|c|c|c|c|c|c|c|c|c|c|c|}
\hline \multirow{3}{*}{$\begin{array}{c}\text { Region } \\
\text { IDs }\end{array}$} & \multirow{3}{*}{ Cities } & \multicolumn{11}{|c|}{ MDCs (total $\mathbf{P M}_{2.5}$ concentrations) } \\
\hline & & \multicolumn{5}{|c|}{ Within SCB } & \multicolumn{4}{|c|}{ Outside SCB } & \multirow{2}{*}{ SOA } & \multirow{2}{*}{ Other* } \\
\hline & & $\mathbf{R} 1$ & $\mathbf{R 2}$ & $\mathbf{R 3}$ & R4 & R5 & R6 & R7 & R8 & R9 & & \\
\hline $\mathbf{R 1}$ & Chongqing & $147(214)$ & $5(80)$ & $6(82)$ & $0(48)$ & $3(87)$ & $4(65)$ & $30(69)$ & $0(48)$ & $2(64)$ & $21(50)$ & $10(42)$ \\
\hline \multirow{5}{*}{$\mathbf{R 2}$} & Bazhong & $4(50)$ & $17(32)$ & $4(32)$ & $2(42)$ & $2(38)$ & $1(38)$ & $36(63)$ & $0(7)$ & $1(39)$ & $23(92)$ & $6(16)$ \\
\hline & Dazhou & $2(49)$ & $43(56)$ & $3(43)$ & $1(42)$ & $3(50)$ & $1(13)$ & $31(65)$ & $0(12)$ & $1(35)$ & $42(214)$ & $6(23)$ \\
\hline & Guangan & $18(88)$ & $40(60)$ & $3(49)$ & $1(39)$ & $3(50)$ & $2(28)$ & $33(71)$ & $0(16)$ & $2(41)$ & $16(49)$ & $7(25)$ \\
\hline & Guangyuan & $7(46)$ & $22(38)$ & $7(38)$ & $3(38)$ & $1(47)$ & $2(43)$ & $23(48)$ & $0(23)$ & $1(47)$ & $23(81)$ & $8(13)$ \\
\hline & Nanchong & $19(84)$ & $45(73)$ & $6(45)$ & $3(42)$ & $2(55)$ & $3(49)$ & $29(80)$ & $0(25)$ & $1(51)$ & $33(88)$ & $7(42)$ \\
\hline \multirow{11}{*}{$\mathbf{R 3}$} & Deyang & $13(54)$ & $3(41)$ & $48(57)$ & 44 (89) & $1(82)$ & $2(78)$ & $18(82)$ & $0(74)$ & $1(30)$ & $19(46)$ & $9(23)$ \\
\hline & Leshan & $7(63)$ & $1(50)$ & 34 (45) & $16(56)$ & $0(66)$ & $3(80)$ & $17(66)$ & $0(80)$ & $1(80)$ & $25(80)$ & $7(24)$ \\
\hline & Luzhou & $46(88)$ & $3(87)$ & $61(87)$ & $2(30)$ & $1(71)$ & $6(47)$ & $24(55)$ & $0(65)$ & $1(47)$ & $29(88)$ & $9(65)$ \\
\hline & Meishan & $9(59)$ & $2(84)$ & $40(55)$ & $55(100)$ & $1(84)$ & $2(74)$ & $21(84)$ & $0(68)$ & $1(68)$ & $25(54)$ & $8(26)$ \\
\hline & Mianyang & $7(57)$ & $4(42)$ & $61(87)$ & $13(85)$ & $1(49)$ & $2(47)$ & $18(64)$ & $0(87)$ & $1(24)$ & $20(81)$ & $10(24)$ \\
\hline & Neijiang & $32(70)$ & $6(44)$ & $61(76)$ & $4(38)$ & $1(104)$ & $5(66)$ & $21(104)$ & $0(41)$ & $1(66)$ & $27(84)$ & $6(29)$ \\
\hline & Suining & $25(53)$ & $18(36)$ & $14(33)$ & $3(33)$ & $2(78)$ & $3(41)$ & $30(53)$ & $0(31)$ & $1(53)$ & $27(81)$ & $7(15)$ \\
\hline & Ya'an & $6(43)$ & $1(23)$ & 13 (39) & $7(22)$ & $0(41)$ & $1(30)$ & $10(37)$ & $0(41)$ & 0 (19) & $28(70)$ & $5(14)$ \\
\hline & Yibin & $12(67)$ & $1(47)$ & $60(73)$ & $4(49)$ & $1(73)$ & $5(87)$ & $19(73)$ & $0(83)$ & $1(34)$ & $18(29)$ & $7(83)$ \\
\hline & Zigong & $21(74)$ & $5(53)$ & $61(76)$ & $3(28)$ & $1(102)$ & $5(80)$ & $21(102)$ & $0(49)$ & $1(80)$ & $27(87)$ & $6(31)$ \\
\hline & Ziyang & $16(56)$ & $3(39)$ & 39 (53) & $21(54)$ & $1(80)$ & $3(56)$ & $21(47)$ & $0(56)$ & $1(56)$ & $23(66)$ & $8(20)$ \\
\hline R4 & Chengdu & $8(55)$ & $2(97)$ & $19(68)$ & $73(112)$ & $1(107)$ & 2 (89) & $21(107)$ & $0(74)$ & $1(27)$ & $26(66)$ & $8(31)$ \\
\hline
\end{tabular}

* Others include IC, BC, windblown dust, and sea salt. 


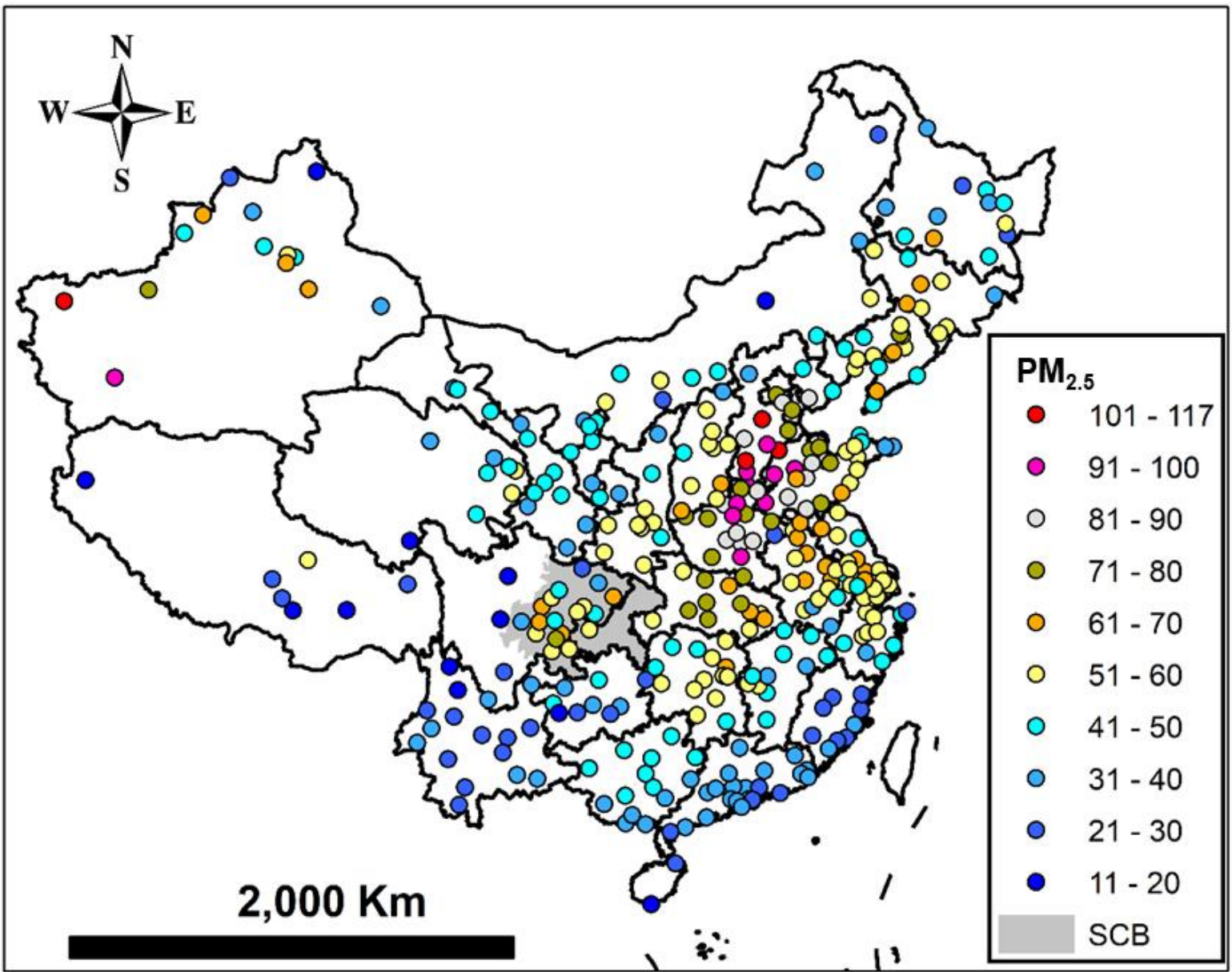

53

Figure S1. Annual average $\mathrm{PM}_{2.5}$ concentrations $\left(\mu \mathrm{g} \mathrm{m}^{-3}\right)$ in major cities of China in 2015. Data source: China National Environmental Monitoring Center, http://106.37.208.233:20035/ 

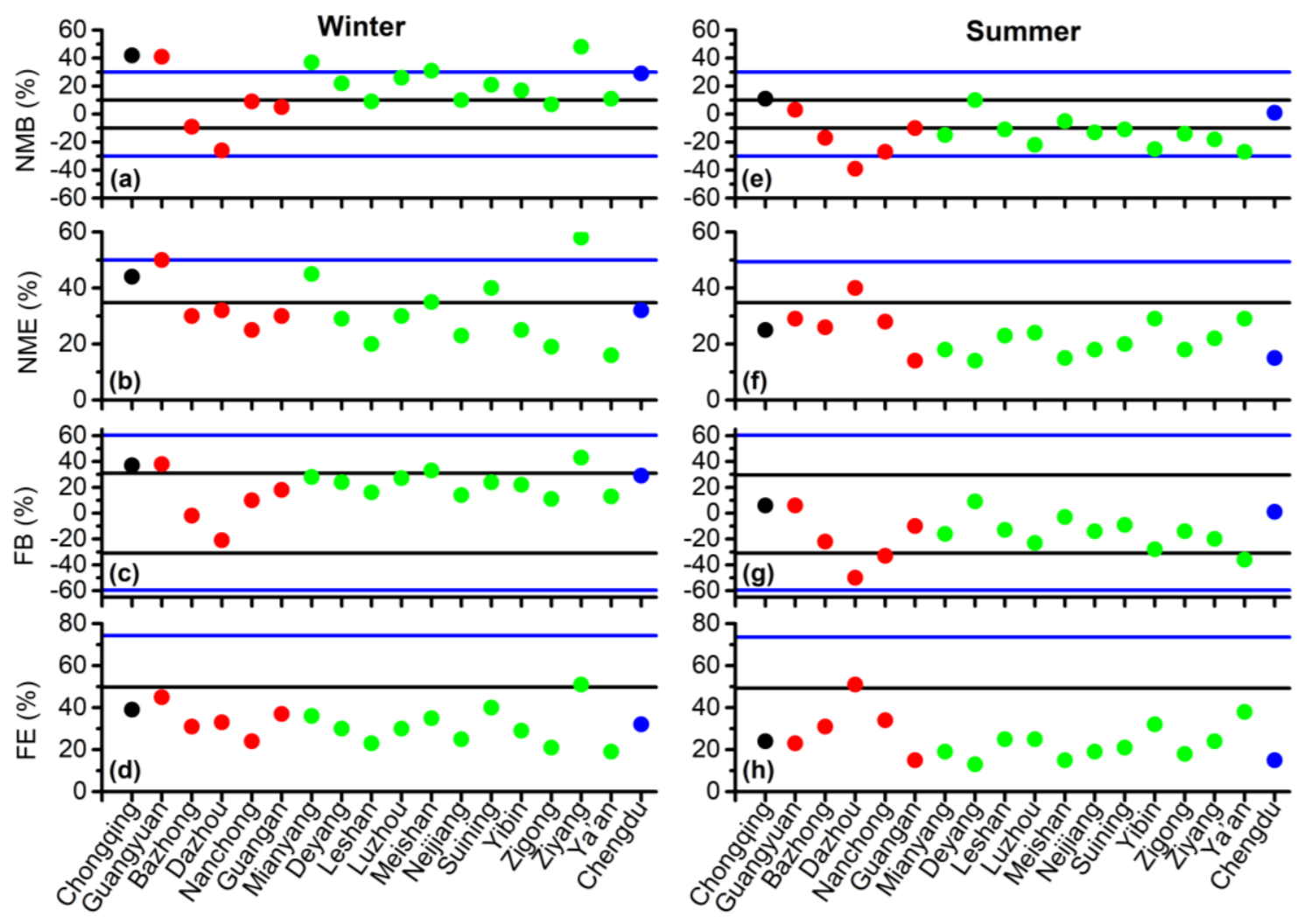

Region ID:

R1

R2 $\bullet$ R3

R4

58

59

60

61

62

63

64

65

Figure S2. Model performance for 24-hr $\mathrm{PM}_{2.5}$ in the 18 city centers of the SCB in the winter and summer. Note: NMB, NME, FB, and FE denote normalized mean bias, normalized mean error, fractional bias, and fractional error, respectively. The blue and black lines represent the modeling criteria and goals suggested by Emery et al. (2017), respectively. The predicted daily $\mathrm{PM}_{2.5}$ concentrations used to calculate NMB, NME, FB, and FE are those closest to the observations within $3 \times 3$ grid cell regions that surround the urban centers. 

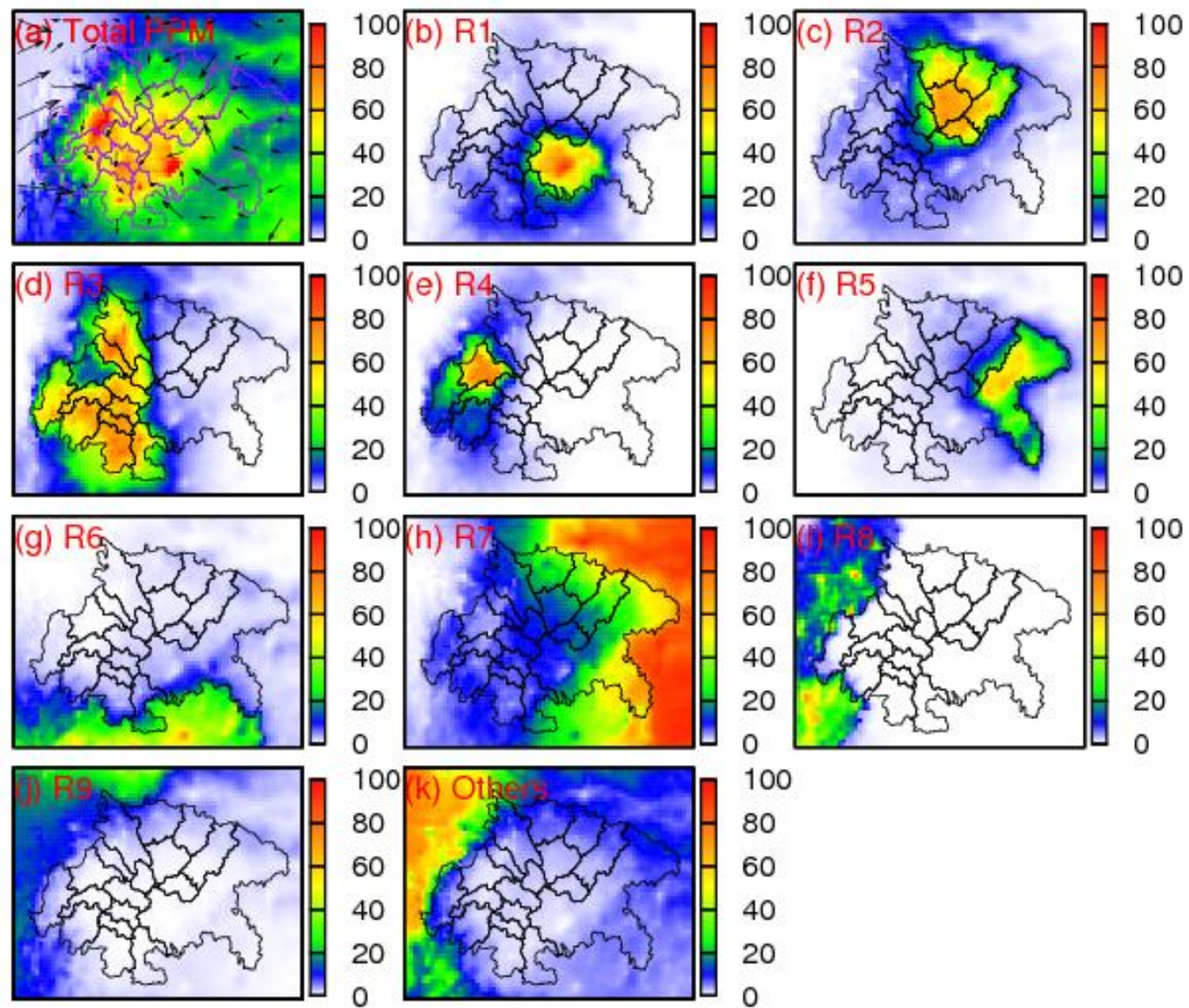

Figure S3. (a) Spatial distributions of predicted PPM concentrations $\left(\mu \mathrm{g} \mathrm{m}^{-3}\right)$ and (b-l) the sourceregion contributions to PPM (\%) in the SCB in the winter. Others include IC, BC, windblown dust, and sea salt. 

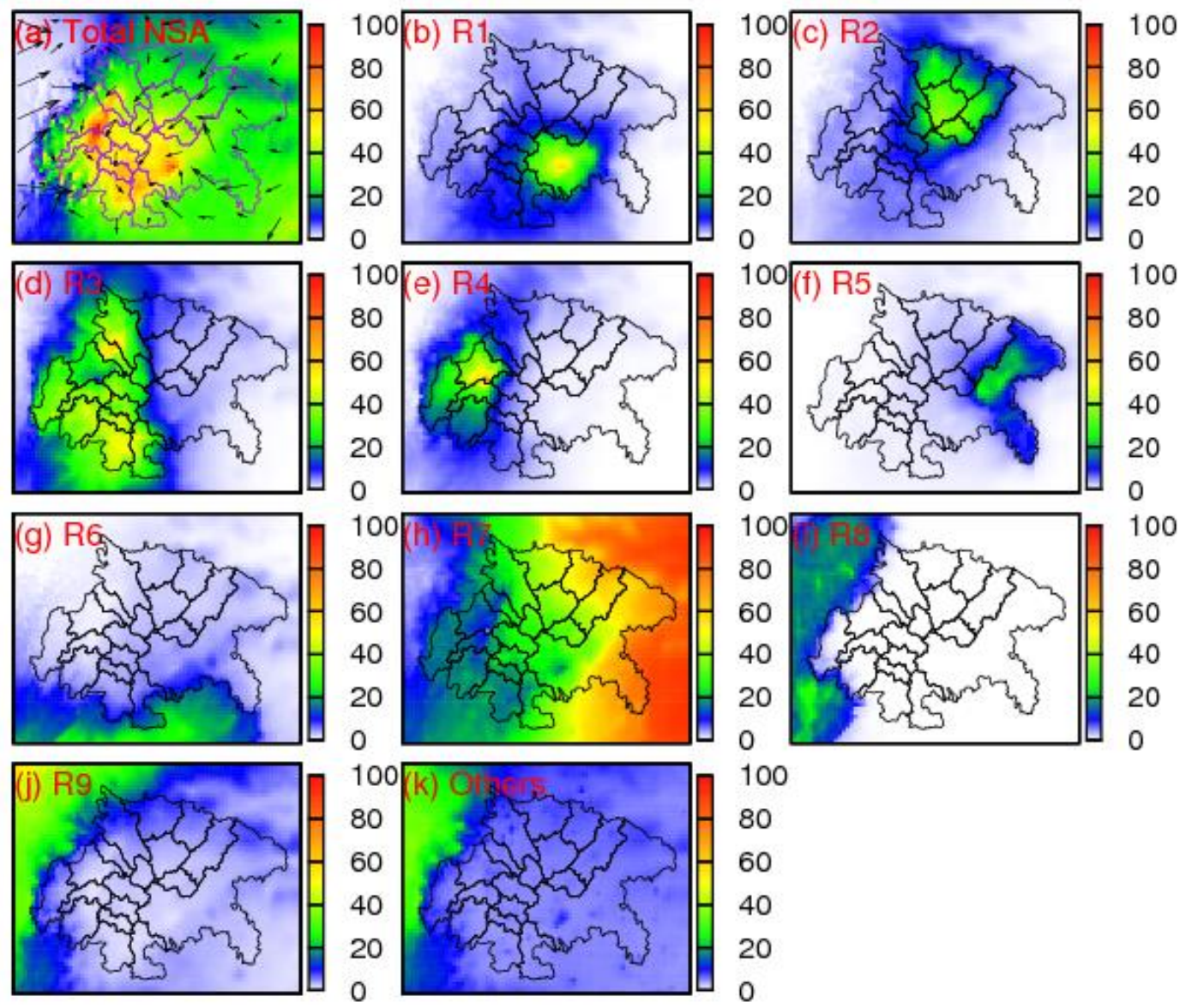

Figure S4. (a) Spatial distributions of predicted seasonal SIA concentrations $\left(\mu \mathrm{g} \mathrm{m}^{-3}\right)$ and (b-l)

76 source-region contributions to SIA (\%)in the SCB in the winter. Others include IC, BC, 77 windblown dust, and sea salt. 

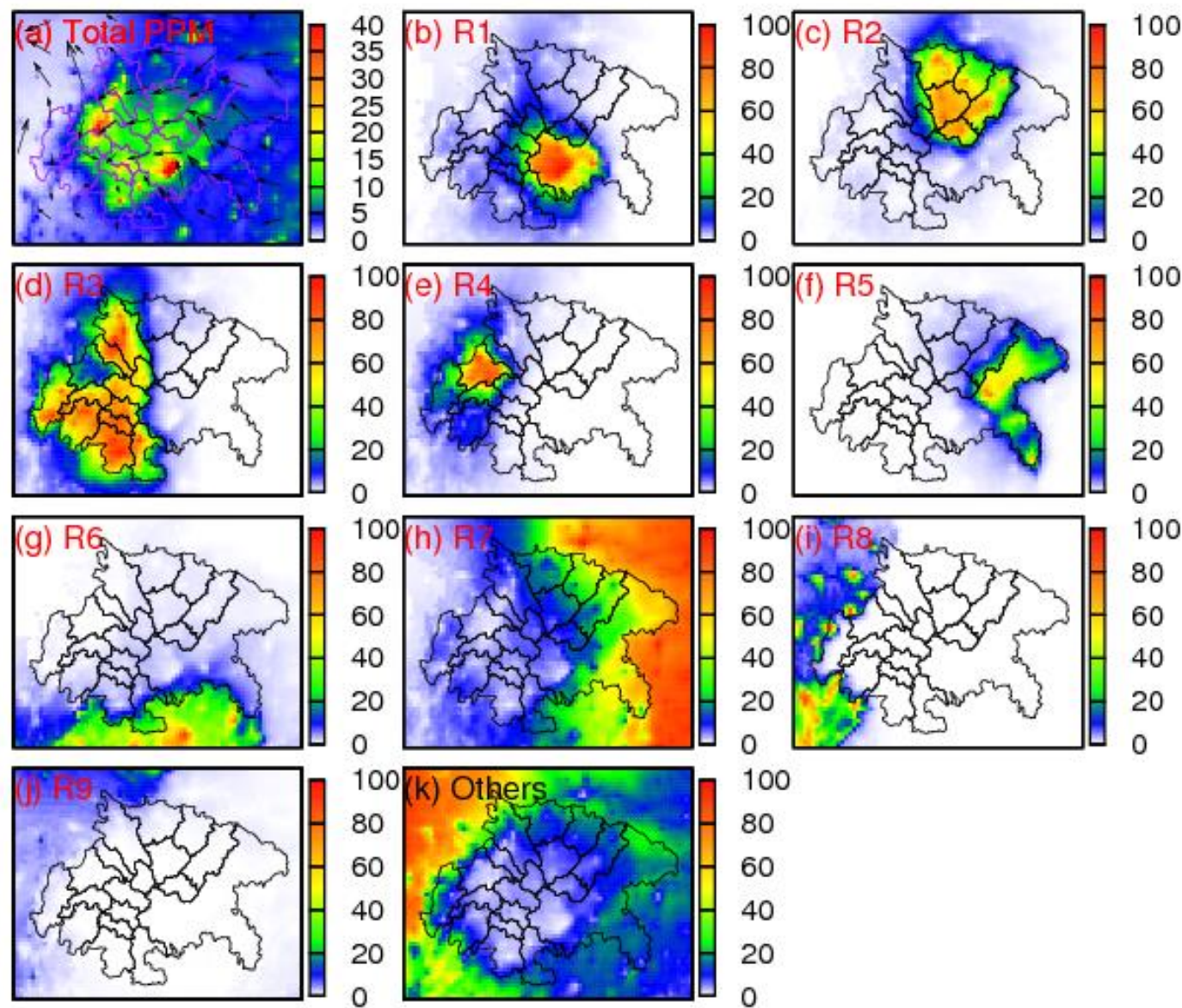

Figure S5. (a) Spatial distributions of predicted PPM concentrations $\left(\mu \mathrm{g} \mathrm{m}^{-3}\right)$ and (b-l) source81 region contributions (\%) to PPM in the SCB in the summer. Others include IC, BC, windblown 82 dust, and sea salt. 

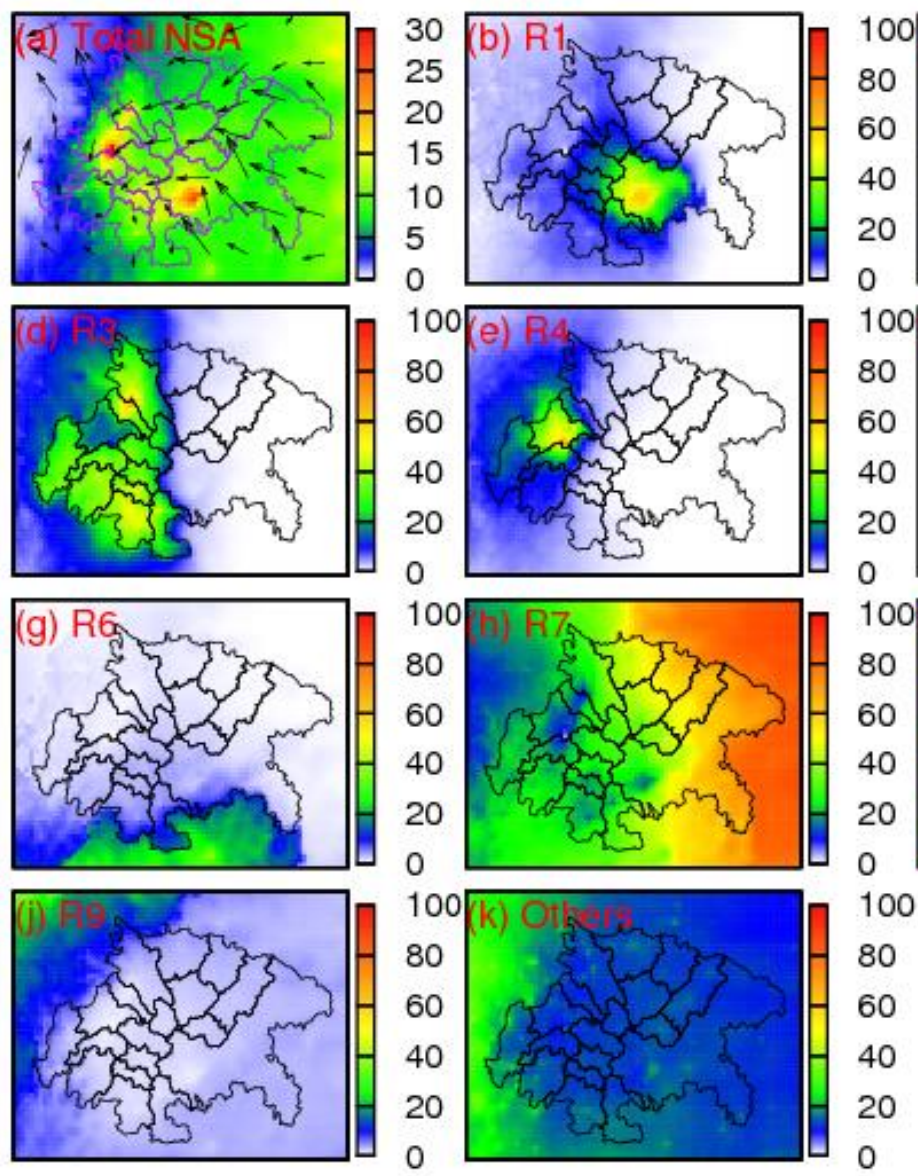
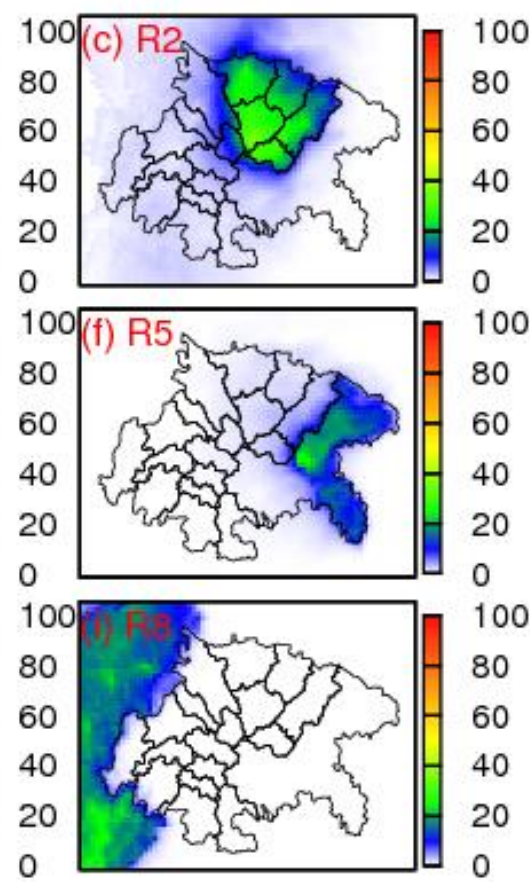

100

80

Figure S6. (a) Spatial distributions of predicted SIA concentrations $\left(\mu \mathrm{g} \mathrm{m}^{-3}\right)$ and (b-1) sourceregion contributions (\%) to SIA in the SCB in the summer. Others include IC, BC, windblown 87 dust, and sea salt. 